\title{
Serum Cystatin C Level as a Biomarker of Aortic Plaque in Patients with an Aortic Arch Aneurysm
}

\author{
Yoshiharu Nishimura, Kentaro Honda, Mitsuru Yuzaki, Kouji Tajima, Ryo Nakamura, Yasuka Nakanishi, \\ Masahiro Kaneko, Kouta Agematsu and Mitsugi Nagashima \\ Department of Thoracic and Cardiovascular Surgery, Wakayama Medical University, Wakayama City, Japan
}

Aim: During surgery for an aortic arch aneurysm, aortic plaque in the descending aorta should be evaluated, but there are currently no suitable biomarkers for it. Surgeons should be especially aware of cerebral embolism from femoral perfusion and of peripheral embolism from stent graft deployment. Cystatin $\mathrm{C}$ is a known useful marker of renal dysfunction with a role as a biomarker for severity of coronary artery disease. In the absence of a suitable biomarker for aortic plaque in the descending aorta, we examine cystatin $\mathrm{C}$ as a candidate.

Methods: In all, 75 patients who underwent surgery for an aortic arch aneurysm were enrolled. They were divided into two groups, depending on whether they had chronic kidney disease or not. The serum cystatin C value and creatinine value were evaluated preoperatively. The aortic plaque volume ratio and components in the descending aorta were calculated from preoperative enhanced computed tomography.

Results: The soft plaque volume ratio was higher in patients with chronic kidney disease than in patients without it. Cystatin $\mathrm{C}$ positively correlated with the total aortic plaque volume ratio in all cases, and it positively correlated with the soft plaque volume ratio in both groups. Creatinine had no correlation with any type of plaque volume ratio in either group. In patients without chronic kidney disease, the soft plaque volume ratio was higher in patients with higher cystatin C levels than in patients with normal levels.

Conclusion: The preoperative serum cystatin $\mathrm{C}$ level could be a biomarker of aortic plaque in the descending aorta in patients with an aortic arch aneurysm.

\section{See editorial vol. 28: 474-476}

Key words: Cystatin C, Biomarker, Aortic plaque, Descending aorta, Aortic arch aneurysm

\section{Introduction}

Cerebral infarction during total arch replacement for an aortic arch aneurysm is a serious postoperative complication $^{1)}$. An important cause is retrograde embolism by aortic plaque in the descending aorta due to femoral perfusion during aortic surgery. This occurs especially in cases of a "shaggy aorta" 2 . The frozen elephant trunk procedure is a recent advance in aortic arch surgery, and it enables quick deployment of a stent graft into the descending aorta. The existence of aortic plaque at the deployment zone of the descending aorta is important in this procedure because it has been shown to potentially lead to para- plegia due to distal embolism to the Adamkiewicz artery $^{3)}$.

Aortic plaque in the descending aorta is mainly diagnosed by enhanced computed tomography, however, computed tomography imagery alone cannot determine the character of aortic plaque, such as its vulnerability. Moreover, biomarkers for aortic plaque have not yet been fully investigated. If there were biomarkers for aortic plaque and its components, the preoperative evaluation of the risk of embolism caused by aortic plaque may be improved.

Cystatin $\mathrm{C}$, an endogenous cysteine protease inhibitor, is known to be a useful marker of renal dysfunction ${ }^{4)}$. It has been reported to have a role as a bio-

Address for correspondence: Yoshiharu Nishimura, Wakayama Medical University, 811-1 Kimiidera, Wakayama 641-8509, Japan

E-mail: nishim-y@wakayama-med.ac.jp

Received: April 15, 2020 Accepted for publication: June 24, 2020

Copyright@2021 Japan Atherosclerosis Society

This article is distributed under the terms of the latest version of CC BY-NC-SA defined by the Creative Commons Attribution License. 
marker for severity of the intima-media thickness of the carotid artery and of coronary artery disease ${ }^{5,6)}$.

In the preoperative evaluation of patients who will undergo cardiovascular surgery, cystatin $\mathrm{C}$ sometimes shows a high value despite normal creatinine levels. In such cases, we hypothesize that cystatin $\mathrm{C}$ might reflect the severity of atherosclerotic plaque of the aorta. Here, to assess whether the serum cystatin $C$ level could be a biomarker for aortic plaque of the descending aorta in patients with an aortic arch aneurysm, we evaluated the relationship between cystatin $\mathrm{C}$ and aortic plaque and its components.

\section{Patients and Methods}

We retrospectively investigated 75 patients with an aortic arch aneurysm who underwent surgery at Wakayama Medical University, Japan between May 2010 and October 2019. This study was approved by the Wakayama Medical University Institutional Review Board (No. 2768), and informed consent was obtained from all the patients. Serum cystatin C and creatinine $(\mathrm{Cr})$ were evaluated preoperatively. The estimated glomerular filtration rate (eGFR) was calculated by the following equation:

eGFR $\left(\mathrm{mL} / \mathrm{min} / 1.73 \mathrm{~m}^{2}\right)=194 \times \mathrm{Cr}^{-1.094} \times \mathrm{age}^{-0.287}$ $\times(0.739$ if female). Chronic kidney disease (CKD) was defined as eGFR $<60 \mathrm{~mL} / \mathrm{min} / 1.73 \mathrm{~m}^{2}$.

Patients were classified into two groups according to the eGFR values: eGFR $<60$ as the CKD group and $\mathrm{eGFR} \geq 60$ as the non-CKD group. Cystatin $\mathrm{C}$ is considered to be a more sensitive indicator of renal dysfunction than creatinine, and it can indicate early stages of renal dysfunction ${ }^{4}$. To clarify the difference between cystatin $\mathrm{C}$ and creatinine-based eGFR, subjects in the non-CKD group were further subdivided into two groups: group A (cystatin $\mathrm{C} \leq$ $0.95 \mathrm{mg} / \mathrm{L}$ ) and group $B($ cystatin $C>0.95 \mathrm{mg} / \mathrm{L}$ ) (Fig. 1).

Preoperative computed tomography angiography (CTA) of the entire aorta was performed as routine clinical care and used for the evaluation of aortic plaque. Omnipaque 240 (Daiichi Sankyo Co., Ltd., Tokyo, Japan) was used as the contrast agent for CTA, and the amount used was $450 \mathrm{mg} \mathrm{I} / \mathrm{kg}$. Analysis of aortic plaque was performed using an Aquarius iNtuition Viewer (TeraRecon Inc., Foster, CA, USA). CTA images were reconstructed in the straightened multiplanar reformatting mode with centerline. The section of the thoracic aorta between the origin of the left subclavian artery and of the celiac artery was isolated as the region of interest (ROI). The program automatically calculates the volume of the aorta. Aortic plaque volume was classified into soft, intermediate, or hard.
By Hounsfield units (HU), soft plaque was defined as -25 to $50 \mathrm{HU}$, intermediate plaque was defined as 51-120 HU, and hard plaque was defined as 501$2000 \mathrm{HU}^{7,8)}$. Total aortic plaque volume was defined as the sum of the volume of soft, intermediate, and hard plaque. The volume of the descending aorta may vary from patient to patient. Therefore, to adjust for this difference, we defined the aortic plaque volume ratio as the percentage of the volume of each component in the total volume of the descending aorta. To differentiate aortic plaque from mural thrombus of the aortic arch aneurysm, if the aortic arch aneurysm involved the origin of the left subclavian artery, ROI was defined as the range from the distal endpoint of the aneurysm to the origin of the celiac artery. Patients with an aortic arch aneurysm combined with a descending aortic aneurysm, and patients with a highly tortuous aorta, were excluded from this study because of difficulty in measurement.

\section{Statistical Analysis}

The distribution of each variable was checked for normality using the Kolmogorov-Smirnov test. Continuous variables were expressed as mean $\pm S D$ or, where indicated, median and interquartile range. Categorical variables were expressed as number and percentages. For continuous variables, statistical differences between the two groups were evaluated by the parametric unpaired $t$-test and the nonparametric Mann-Whitney $U$ test. For categorical data, the twogroup comparison was evaluated by the $\chi^{2}$ test. Correlation analysis between biomarker and aortic plaque volume ratio was evaluated by Pearson's correlation for parametric variables and Spearman's correlation for nonparametric variables. Spearman's $\rho$ was used as the correlation coefficient. Statistical results were reported as significant if $P<0.05$. Statistical analysis was performed using Stat Mate III (ATMS Co., Ltd., Chiba, Japan).

\section{Results}

Patient characteristics, classified into the CKD group and the non-CKD group by renal function, are shown in Table 1. There were no significant differences in age, gender, total cholesterol value, triglyceride value, or low-density or high-density lipoprotein cholesterol value between the two groups. Combination with coronary artery disease (CAD) or carotid artery stenosis (CAS) was more common in the CKD group, but combination with peripheral artery disease, diabetes mellitus or an abdominal aortic aneurysm, and history of smoking was similar between the two groups. 
Table 1. Subject Characteristics

\begin{tabular}{|c|c|c|c|c|}
\hline & $\begin{array}{c}\text { All } \\
(n=75)\end{array}$ & $\begin{array}{l}\text { CKD group } \\
(n=48)\end{array}$ & $\begin{array}{l}\text { non-CKD group } \\
\qquad(n=27)\end{array}$ & $P$ value \\
\hline Age (year) & $74.5 \pm 6.4$ & $74.9 \pm 5.2$ & $74.0 \pm 8.1$ & 0.610 \\
\hline Gender (male/female) & $57 / 18$ & $37 / 11$ & $20 / 7$ & 0.707 \\
\hline CAD (\%) & 31 & 40 & 15 & 0.048 \\
\hline PAD (\%) & 16 & 19 & 11 & 0.591 \\
\hline CAS (\%) & 16 & 25 & 0 & 0.012 \\
\hline AAA (\%) & 37 & 35 & 41 & 0.647 \\
\hline DM $(\%)$ & 7 & 8 & 4 & 0.440 \\
\hline Smoking (\%) & 63 & 63 & 63 & 0.968 \\
\hline Cystatin C (mg/L) & $1.21 \pm 0.39$ & $1.34 \pm 0.40$ & $0.95 \pm 0.19$ & $<0.001$ \\
\hline Creatinine (mg/dL) & $1.04 \pm 0.35$ & $1.19 \pm 0.36$ & $0.78 \pm 0.02$ & $<0.001$ \\
\hline $\mathrm{eGFR}\left(\mathrm{ml} / \mathrm{min} / 1.73 \mathrm{~m}^{2}\right)$ & $55.1 \pm 14.6$ & $46.5 \pm 9.0$ & $70.3 \pm 9.0$ & $<0.001$ \\
\hline Total Chol (mg/dL) & $194 \pm 43$ & $192 \pm 47$ & $196 \pm 34$ & 0.714 \\
\hline Triglyceride (mg/dL) & $143 \pm 63$ & $142 \pm 67$ & $144 \pm 58$ & 0.890 \\
\hline LDL Chol (mg/dL) & $115 \pm 36$ & $112 \pm 41$ & $119 \pm 27$ & 0.381 \\
\hline HDL Chol (mg/dL) & $46 \pm 12$ & $46 \pm 12$ & $47 \pm 12$ & 0.545 \\
\hline
\end{tabular}

CAD: coronary artery disease, PAD: peripheral artery disease, CAS: carotid artery stenosis

AAA: abdom inal aortic aneurysm, DM: diabetes mellitus, Chol: cholesterol $P$ value: CKD group vs non CKD group

Table 2. Total aortic plaque volume ratio and its component

\begin{tabular}{|c|c|c|c|c|}
\hline & $\begin{array}{c}\text { All } \\
(n=75)\end{array}$ & $\begin{array}{l}\text { CKD group } \\
\quad(n=48)\end{array}$ & $\begin{array}{l}\text { non-CKD group } \\
\qquad(n=27)\end{array}$ & $p$ value \\
\hline Total aortic plaque (\%) & $19.4 \pm 5.5$ & $30.4 \pm 5.7$ & $28.3 \pm 3.4$ & 0.066 \\
\hline Intermediate plaque (\%) & $9.6 \pm 2.6$ & $9.7 \pm 2.8$ & $9.3 \pm 2.3$ & 0.531 \\
\hline Hard plaque (\%) & $0.13(0.03-0.29)$ & $0.15(0.07-0.33)$ & $0.10(0.02-0.20)$ & 0.082 \\
\hline
\end{tabular}

$p$ value: CKD group vs non CKD group

Table 3. Correlation between cystatin $\mathrm{C}$ and plaque volume ratio

\begin{tabular}{|c|c|c|c|c|c|c|}
\hline & \multicolumn{2}{|c|}{$\operatorname{ALL}(n=75)$} & \multicolumn{2}{|c|}{ CKD group $(n=48)$} & \multicolumn{2}{|c|}{ non-CKD group $(n=27)$} \\
\hline & Coefficient & $P$ value & Coefficient & $P$ value & Coefficient & $P$ value \\
\hline Total aortic plaque (\%) & $r=0.266$ & 0.021 & $\rho=0.226$ & NS & $r=0.397$ & 0.040 \\
\hline Soft plaque (\%) & $r=0.345$ & 0.002 & $\rho=0.318$ & $<0.05$ & $r=0.417$ & 0.030 \\
\hline Intermediate plaque $(\%)$ & $r=0.122$ & 0.297 & $\rho=0.065$ & NS & $r=0.296$ & 0.134 \\
\hline
\end{tabular}

Table 2 shows the total aortic plaque volume ratio and its components in all cases and in cases with or without CKD. The soft plaque volume ratio was significantly higher in the CKD group than in the non-CKD group.

The correlation between cystatin $\mathrm{C}$ and plaque volume ratio is shown in Table 3. The cystatin $\mathrm{C}$ level correlated positively with the total aortic plaque volume ratio, soft plaque volume ratio, and hard plaque volume ratio in all cases. In terms of renal function, the cystatin $\mathrm{C}$ level correlated better with the soft plaque volume ratio in the $\mathrm{CKD}$ group. In the non$\mathrm{CKD}$ group, the cystatin $\mathrm{C}$ level correlated best with the soft plaque volume ratio. Cystatin $\mathrm{C}$ also correlated with the total aortic plaque volume ratio in the non-CKD group.

The correlation between creatinine and the plaque volume ratio is shown in Table 4 . The creati- 
Table 4. Correlation between creatinine and plaque volume ratio

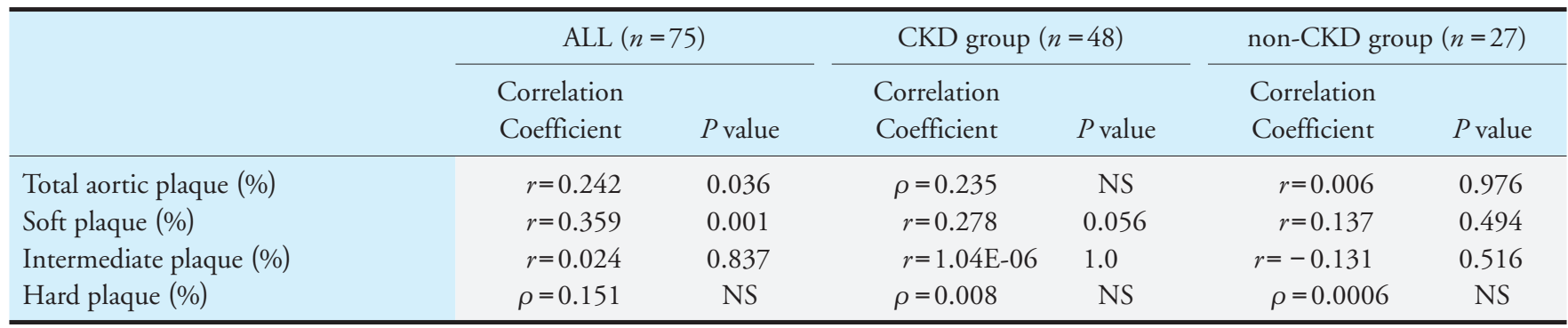

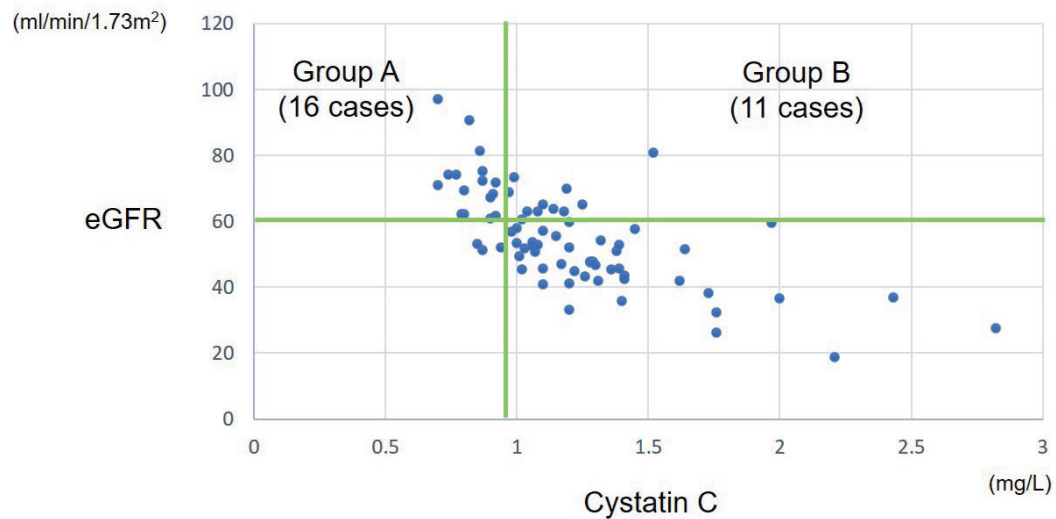

Fig. 1. Higher cystatin level in the non-CKD group

Group B showed a higher level of cystatin C among groups in which eGFR was maintained at $60 \mathrm{~mL} / \mathrm{min} / 1.73 \mathrm{~m}^{2}$ or more.

Table 5. High cystatin C level despite normal renal function

\begin{tabular}{lccc}
\hline & Group A $(n=16)$ & Group B $(n=11)$ & $P$ value \\
\hline Total aortic plaque (\%) & $16.6 \pm 0.8$ & $19.5 \pm 1.3$ & 0.080 \\
Soft plaque (\%) & $7.5 \pm 1.9$ & $9.2 \pm 2.2$ & 0.034 \\
Intermediate plaque (\%) & $8.9 \pm 1.9$ & $10.0 \pm 2.7$ & 0.241 \\
Hard plaque (\%) & $0.09(0.02-0.27)$ & $0.10(0.01-0.16)$ & NS \\
\hline
\end{tabular}

Group A: Cystatin C<0.95, eGFR $>60$

Group B: Cystatin C >0.95, eGFR > 60

nine level correlated positively with the total aortic plaque volume and soft plaque in all cases. There were no significant correlations, however, with the total aortic plaque volume ratio or its components in either the CKD group or the non-CKD group.

As mentioned in the patients and methods section, to clarify the difference between cystatin $\mathrm{C}$ and creatinine-based eGFR, subjects in the non-CKD group were further subdivided into two groups: group A (cystatin $\mathrm{C} \leq 0.95 \mathrm{mg} / \mathrm{L}$ ) and group $\mathrm{B}$ (cystatin $\mathrm{C}$ $>0.95 \mathrm{mg} / \mathrm{L})$ (Fig. 1). Table 5 shows the total aortic plaque volume ratio and its components in groups $\mathrm{A}$ and $\mathrm{B}$; the soft plaque volume ratio was higher in group B than in group A.
Preoperative laboratory data, including total cholesterol, triglyceride, and high-density/low-density lipoprotein cholesterol, did not positively correlate with the total aortic plaque volume ratio or its components.

In relation to cystatin $\mathrm{C}$ and atherosclerosis, $\mathrm{CAD}$ and CAS as co-morbidities have been reported to be associated with higher serum cystatin $\mathrm{C}$ levels ${ }^{9)}$. In addition, CAD and CAS were significantly different between the CKD group and the non-CKD group among the subjects (Table 1). We therefore considered that CAD and CAS could be confounding factors in the association between cystatin $\mathrm{C}$ and aortic plaque, and conducted the same study in 47 cases 
Table 6. Correlation between cystatin $\mathrm{C}$ and plaque volume ratio in patients without coronary artery disease and carotid artery stenosis

\begin{tabular}{|c|c|c|c|c|c|c|}
\hline & \multicolumn{2}{|c|}{$\begin{array}{l}\text { Patient without CAD and CAS } \\
\qquad(n=47)\end{array}$} & \multicolumn{2}{|c|}{$\begin{array}{l}\text { CKD group } \\
\quad(n=24)\end{array}$} & \multicolumn{2}{|c|}{$\begin{array}{l}\text { non-CKD group } \\
\qquad(n=23)\end{array}$} \\
\hline & $\begin{array}{l}\text { Correlation } \\
\text { Coefficient }\end{array}$ & $P$ value & $\begin{array}{l}\text { Correlation } \\
\text { Coefficient }\end{array}$ & $P$ value & $\begin{array}{l}\text { Correlation } \\
\text { Coefficient }\end{array}$ & $P$ value \\
\hline Total aortic plaque (\%) & $r=0.235$ & 0.112 & $r=0.111$ & 0.604 & $r=0.451$ & 0.031 \\
\hline Soft plaque (\%) & $r=0.334$ & 0.022 & $r=0.292$ & 0.167 & $r=0.436$ & 0.038 \\
\hline
\end{tabular}

CAD: coronary artery disease, CAS: carotid artery stenosis

Table 7. Correlation between creatinine and plaque volume ratio in patients without coronary artery disease and carotid artery stenosis

\begin{tabular}{|c|c|c|c|c|c|c|}
\hline & \multicolumn{2}{|c|}{$\begin{array}{l}\text { Patient without CAD and CAS } \\
\qquad(n=47)\end{array}$} & \multicolumn{2}{|c|}{$\begin{array}{l}\text { CKD group } \\
\quad(n=24)\end{array}$} & \multicolumn{2}{|c|}{$\begin{array}{l}\text { non-CKD group } \\
\qquad(n=23)\end{array}$} \\
\hline & $\begin{array}{l}\text { Correlation } \\
\text { Coefficient }\end{array}$ & $P$ value & $\begin{array}{l}\text { Correlation } \\
\text { Coefficient }\end{array}$ & $P$ value & $\begin{array}{l}\text { Correlation } \\
\text { Coefficient }\end{array}$ & $P$ value \\
\hline Total aortic plaque (\%) & $r=0.094$ & 0.532 & $r=0.006$ & 0.979 & $r=-0.0004$ & 0.998 \\
\hline Soft plaque (\%) & $r=0.182$ & 0.220 & $r=0.131$ & 0.543 & $r=0.147$ & 0.502 \\
\hline Hard plaque (\%) & $\rho=0.080$ & NS & $\rho=-0.070$ & NS & $\rho=0.024$ & NS \\
\hline
\end{tabular}

CAD: coronary artery disease, CAS: carotid artery stenosis

Table 8. High cystatin $\mathrm{C}$ level despite normal renal function in patients without coronary artery disease and carotid artery stenosis

\begin{tabular}{lccc}
\hline & Group A $(n=16)$ & Group B $(n=7)$ & $P$ value \\
\hline Total aortic plaque (\%) & $16.6 \pm 3.2$ & $20.2 \pm 4.9$ & 0.113 \\
Soft plaque (\%) & $7.5 \pm 1.8$ & $9.5 \pm 2.1$ & 0.032 \\
Intermediate plaque (\%) & $8.9 \pm 1.9$ & $10.6 \pm 3.1$ & 0.215 \\
Hard plaque (\%) & $0.09(0.02-0.27)$ & $0.10(0.06-0.14)$ & NS \\
\hline
\end{tabular}

Group A: Cystatin C $<0.95$, eGFR $>60$

Group B: Cystatin C > 0.95, eGFR > 60

after the exclusion of subjects with CAD and CAS (Tables 6-8). Cystatin C significantly correlated with the soft plaque volume ratio in patients without $\mathrm{CAD}$ and CAS. In the non-CKD group, cystatin $\mathrm{C}$ significantly correlated with the total aortic plaque volume ratio and soft plaque volume ratio (Table 6). Creatinine did not show any correlation with aortic plaque volume and its components in patients without CAD and CAS (Table 7). In patients who had higher cystatin $\mathrm{C}$ levels in the non-CKD group without $\mathrm{CAD}$ and CAS, the soft plaque volume ratio was higher than in patients who had normal cystatin C levels (Table 8).

Within this study cohort, we did not observe postoperative cerebral infarction or distal thromboem- bolism in clinical practice, including spinal ischemia and intestinal ischemia.

\section{Discussion}

We investigated biomarkers for aortic plaque in the descending aorta of patients with an aortic arch aneurysm. Preoperative serum cystatin $C$ levels were found to be correlated with aortic plaque volume in the descending aorta and also with soft plaque volume by components. If patients with CAD and CAS were excluded, the preoperative cystatin $\mathrm{C}$ level also correlated with soft plaque volume.

To date, biomarkers for aortic plaque have not been widely investigated. Momiyama et al. reported 
that plasma high-sensitivity C-reactive protein (hsCRP) correlates with aortic plaque using the plaque score based on aortic magnetic resonance imaging slices ${ }^{10)}$. Agmon et al. reported the association between the hsCRP level and the presence of aortic plaque using three segments of images of intimal thickness by transesophageal echocardiography ${ }^{11)}$. Regarding cystatin $\mathrm{C}$ as a biomarker of atherosclerosis, Sugiyama et $a l$. reported that serum cystatin $\mathrm{C}$ levels are associated with coronary artery calcification examined by multidetector computed tomography ${ }^{5}$. Kaneko et al. reported that the serum cystatin $C$ level was associated with carotid arterial elasticity ${ }^{6}$. To the best of our knowledge, however, there have been no reports on the relationship between cystatin $\mathrm{C}$ and aortic plaque and its components.

It can be speculated that there are two possible mechanisms by which cystatin $\mathrm{C}$ correlates with total aortic volume and soft plaque volume: the effect of renal dysfunction on aortic plaque, and the direct effect of cystatin $\mathrm{C}$ on aortic plaque aside from renal dysfunction.

\section{Association between Atherosclerotic Plaque and Cystatin C Reflecting Renal Dysfunction}

In the present study, the soft plaque volume ratio was higher in the CKD group than in the non-CKD group. Both the cystatin $\mathrm{C}$ level and creatinine level showed a positive correlation with the soft plaque volume ratio in all cases. These results suggest that renal dysfunction itself may promote the development of soft plaque in the descending aorta. Amann et al. reported that uremia may affect the wall thickness of the aorta in experimental renal failure ${ }^{12)}$. The presence of CKD was reported by Matsumura et al. to be associated with complex aortic plaques detected using transesophageal echocardiography, and they suggested that this association is stronger in the descending aorta than in the proximal aorta ${ }^{13)}$. As a possible mechanism, they suggested embolization of the plaque from the descending aorta to renal vasculature, or an association between CKD and systemic atherosclerosis.

In our cohort, however, when patients were divided into $\mathrm{CKD}$ and non-CKD groups, cystatin $\mathrm{C}$ and creatinine showed different correlations with the soft plaque volume ratio. The cystatin $\mathrm{C}$ level showed a positive correlation with the soft plaque volume ratio in both the CKD and non-CKD groups in all cases, but the creatinine level did not. These results suggest that cystatin $\mathrm{C}$ and creatinine, which are both indicators of renal function, differ as biomarkers for aortic plaque. To clarify this difference, we evaluated the cases in which serum cystatin $\mathrm{C}$ shows a high value despite retention of renal function (creatinine-based
eGFR $\geq 60 \mathrm{~mL} / \mathrm{min} / 1.73 \mathrm{~m}^{2}$ ), and we found that a higher cystatin $\mathrm{C}$ level in the non-CKD group was associated with a greater soft plaque volume ratio. This may be because cystatin $\mathrm{C}$ reflects early renal dysfunction better than creatinine-based eGFR. Similar reports have been made regarding CAD. Shlipak et al. reported that cystatin $\mathrm{C}$, which reflects the state of preclinical kidney disease, is a prognostic biomarker of risk for cardiovascular disease among elderly persons without $\mathrm{CKD}^{14)}$. Imai et al. reported that cystatin $\mathrm{C}$ concentrations were correlated with early-stage coronary atherosclerotic plaque among patients without established chronic kidney dysfunction. In their report, the mechanism of this association was assumed to represent early kidney disease ${ }^{15)}$.

Effect of Cystatin C on Atherosclerotic Plaque other than Renal Dysfunction

Regarding the difference between the effects of cystatin $\mathrm{C}$ and creatinine on aortic plaque, effects other than renal dysfunction may be a consideration. Koenig et al. reported that cystatin $\mathrm{C}$ concentrations have a strong association with secondary cardiovascular events in patients with $\mathrm{CAD}^{16)}$. They speculated that plasma cystatin $\mathrm{C}$ increased to compensate for the increased activity of elastolytic cysteine protease stimulated by inflammatory cytokines during vascular injury. Batra et al. reported that higher plasma cystatin C levels were associated with a higher carotid intimamedia thickness, and in patients with normal or nearnormal renal function, higher cystatin $\mathrm{C}$ levels were associated with severity of $\mathrm{CAD}^{17}$. They postulated that the association between cystatin $\mathrm{C}$ and cardiovascular events may be due either to direct pathological effects or to a heightened inflammatory state and clustering with other risk factors. Regarding the direct pathological effect of cystatin C for the aorta, Shi et al. reported that cystatin $\mathrm{C}$ expression decreased in the wall of an abdominal aortic aneurysm ${ }^{18)}$. Conversely, elastolytic cathepsin, for which cystatin $\mathrm{C}$ is an inhibitor, is overexpressed both in the atherosclerotic plaque and in the wall of the abdominal aortic aneurysm. Abisi et al. investigated the activity of cathepsin and cystatin $\mathrm{C}$ in the wall of the abdominal aortic aneurysm and aortic occlusive disease. They found that the cystatin C levels were significantly lower in the wall of the abdominal aortic aneurysm compared with the levels in aortic occlusive disease ${ }^{19)}$. Salgado et al. reported on the discrepancy between tissue and circulating cystatin $\mathrm{C}$ levels. They speculated that higher serum cystatin $\mathrm{C}$ would result from cytokine-stimulated cells, which release cystatin $\mathrm{C}$ into circulation during atherosclerosis and compensate for the decreased cystatin $\mathrm{C}$ in the plaque ${ }^{\text {9) }}$. They also sug- 
gested that cystatin $\mathrm{C}$ has different roles in circulation and in the plaque.

\section{Cystatin C and Plaque Vulnerability}

In the current study, cystatin $\mathrm{C}$ had notable correlation with soft plaque among the plaque components. Soft plaque can be considered as unstable, vulnerable, or noncalcified. Zhao et al. reported that cystatin $\mathrm{C}$ was downregulated in the unstable plaque of the popliteal artery ${ }^{20)}$. The interplay between cathepsin and cystatin $\mathrm{C}$ may underlie the progression of plaques from stable to unstable. Regarding the association between cystatin $\mathrm{C}$ and plaque vulnerability, Dai et al. reported that cystatin C-based eGFR is related to a higher prevalence of vulnerable plaque of CAD by optical coherence tomography compared with creatinine-based eGFR ${ }^{21)}$. Serum cystatin $\mathrm{C}$ concentrations were reported by Imai et al. to be more strongly associated with noncalcified plaques in CAD detected by multidetector computed tomography ${ }^{15)}$.

Whatever the mechanism, our results suggest that in patients with an aortic arch aneurysm with near-normal renal function, higher cystatin $\mathrm{C}$ levels are associated with soft plaque volume in the descending aorta. Therefore, if significant plaque is detected in the descending aorta by preoperative CTA in patients with an aortic arch aneurysm, serum cystatin $\mathrm{C}$ may be used as a biomarker. If the serum cystatin C levels are high, it is suggested that there will be a larger quantity of soft plaques in the descending aorta. In such cases, a surgical strategy should be considered, including the site of arterial perfusion selection, with the assumption that aortic plaque in the descending aorta is vulnerable.

\section{Study Limitation}

We used the correlation coefficient as a statistical analysis in this study. There is no way to adjust the confounding factor for the correlation coefficient statistically; therefore, subgroup analyses excluding CAD and CAS were performed as an alternative method. Owing to the small number of cases, however, the effects of age and sex on cystatin $\mathrm{C}$ could not be adjusted. In this study, it was not possible to examine the clinical effect of the relationship between serum cystatin $\mathrm{C}$ levels and aortic plaque on perioperative complications during total arch replacement for an aortic arch aneurysm. Moreover, the number of cases was small, and further evaluation is needed.

\section{Conclusion}

Preoperative serum cystatin C levels could be a biomarker for aortic plaque in the descending aorta in patients with an aortic arch aneurysm. The effect of cystatin $\mathrm{C}$ on the progression of soft plaque volume was suggested by both the effect of renal dysfunction and the direct effect of cystatin $\mathrm{C}$ on atherosclerosis.

\section{Acknowledgements}

We acknowledge proofreading and editing by Benjamin Phillis at the Clinical Study Support Center, Wakayama Medical University.

\section{Notice of Grant}

This study was financially supported by research funding from KAKENHI.

\section{Conflicts of Interest}

This study was financially supported by research funding from KAKENHI. There are no other conflicts of interest to declare.

\section{References}

1) Shiiya N: Aortic arch replacement for degenerative aneurysm: advances during the last decade. Gen Thorac Cardiovasc Surg, 2013; 61: 191-196

2) Sabik JF, Lytle BW, McCarthy PM, Cosgrove DM: Axillary artery: an alternative site of arterial cannulation for patients with extensive aortic and peripheral vascular disease. J Thorac Cardiovasc Surg, 1995; 109: 885-891

3) Koizumi S, Nagasawa A, Koyama T: Total aortic arch replacement using frozen elephant trunk technique with J graft open stent graft for distal arch aneurysm. Gen Thorac Cardiovasc Surg, 2018; 66: 91-94

4) Taglieri N, Koenig W, Kaski JC: Cystatin C and cardiovascular risk. Clin Chem, 2009; 55: 1932-1943

5) Sugiyama H, Miyoshi T, Osawa K, Miki T, Koide Y, Nakamura K, Morita H, Ito H: Serum cystatin C levels are associated with coronary artery calcification in women without chronic kidney disease. J Cardiol, 2017; 70: 559564

6) Kaneko R, Sawada S, Tokita A, Honkura R, Tamura N, Kodama S, Izumi T, Takahashi K, Uno K, Imai J, Yamada T, Miyachi Y, Hasegawa H, Kanai H, Ishigaki Y, Katagiri $\mathrm{H}$ : Serum cystatin C level is associated with carotid arterial wall elasticity in subjects with type 2 diabetes mellitus: A potential marker of early-stage atherosclerosis. Diabetes Res Clin Pract, 2018; 139: 43-51

7) Schroeder S, Kopp AF, Baumbach A, Meisner C, Kuettner A, Georg C, Ohnesorge B, Herdeg C, Claussen CD, Karsch KR: Noninvasive detection and evaluation of atherosclerotic coronary plaques with multislice computed tomography. J Am Coll Cardiology, 2001; 37: 1430-1435

8) Fujii T: MDCT no Yuuyousei. INNERVISION, 2008; 23: 4-11

9) Salgado JV, Souza FL, Salgado BJ: How to understand the association between cystatin $\mathrm{C}$ levels and cardiovascular 
disease: Imbalance, counterbalance, or consequence? J Cardiol, 2012; 62: 331-335

10) Momiyama Y, Ohmori R, Fayad ZA, Kihara T, Tanaka N, Kato R, Taniguchi H, Nagata M, Nakamura H, Ohsuzu F: Association between plasma C-reactive protein levels and the severities of coronary and aortic atherosclerosis. J Atheroscler Thromb, 2010; 17: 460-467

11) Agmon Y, Khandheria BK, Meissner I, Petterson TM, O'Fallon WM, Wiebers DO, Christianson TJH, McConnel JP, Whisnant JP, Seward JB, Tajik AJ: C-reactive protein and atherosclerosis of the thoracic aorta: a population-based transesophageal echocardiographic study. Arch Intern Med, 2004; 16: 1787-1781

12) Amann K, Wolf B, Nichols C, Törnig J, Schwarz U, Zeier M, Mall G, Ritzthe E: Aortic changes in experimental renal failure. Hypertension, 1997; 29: 770-775

13) Matsumura Y, Sugioka K, Fujita S, Ito A, Iwata S, Yoshiyama M: Association between chronic kidney disease and thoracic aortic atherosclerosis detected using transesophageal echocardiography. Atherosclerosis, 2014; 237: 301306

14) Shlipak MG, Katz R, Sarnak MJ, Fried LF, Newman AB, Stehman-Breen C, Seliger SL, Kestenbaum B, Psaty B, Tracy RP, Siscivick DS: Cystatin C and prognosis for cardiovascular and kidney outcomes in elderly persons without chronic kidney disease. Ann Intern Med, 2006; 145: 237-246

15) Imai A, Komatsu S, Ohara T, Kamata T, Yoshida J, Miyaji K, Shimizu Y, Takewa M, Hirayama A, Deshpande GA, Takahahsi O, Kodama K: Serum cystatin C is associated with early stage of coronary atherosclerotic plaque morphology on multidetector computed tomography. Atherosclerosis, 2011; 218: 350-355

16) Koenig W, Twardella D, Brenner H, Rothenbacher D: Plasma concentrations of cystatin $\mathrm{C}$ in patients with coronary heart disease and risk for secondary cardiovascular events: More than simply a marker of glomerular filtration rate. Clin Chem, 2005; 51: 321-327

17) Batra A, Kapoor A, Sharma RK, Agrawal N, Sinha A, Kumar S, Garg N, Tewari S, Goel PK: Association of plasma cystatin $\mathrm{C}$ level with angiographically documented coronary artery disease inpatients of Indian origin. J Cardiol, 2012; 59: 182-189

18) Shi G-P, Sukhova GK, Grubb A, Ducharme A, Rhode LH, Lee RT, Ridker PM, Libby P, Chapman HA: Cystatin $\mathrm{C}$ deficiency in human atherosclerosis and aortic aneurysm. J Clin Invest, 1999; 104: 1191-1197

19) Abisi S, Burnand KG, Waltham M, Humphries J, Taylor PR, Smith A: Cysteine protease activity in the wall of abdominal aortic aneurysm. J Vas Surg, 2007; 46: 12601266

20) Zhao H, Qin X, Wang S, Sun X, Dong B: Increased cathepsin $\mathrm{K}$ levels in human atherosclerotic plaque $s$ are associated with plaque instability. Exp Ther Med, 2017; 14: 3471-3476

21) Dai J, Xing L, Hou J, Jia H, Hu S, Tian J, Lin L, Li L, Zhu Y, Zheng G, Zhang S, Yu B, Jang I-K: Chronic kidney disease predicts coronary plaque vulnerability: an optical coherence tomography study. Coron Artery Dis, 2017; 28: 135-144 